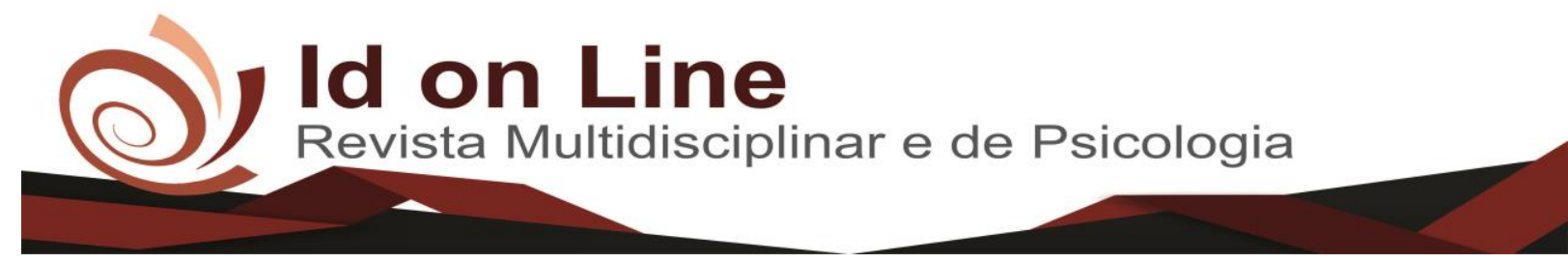

Artigo

\title{
Desafios no atendimento ao paciente portador de necessidades especiais em uma clínica escola
}

\author{
Bianca Cardoso Barros ${ }^{1}$; Daniela Porto da Cunha ${ }^{2}$
}

\begin{abstract}
Resumo: Existe um grande número de pacientes com deficiência que procuram por atendimento odontológico que apesar de possuírem algumas limitações, sejam elas físicas ou psíquicas, possuem direito a inclusão. Atender a essa demanda requer um maior preparo profissional necessitando realizar um atendimento mais humanizado. A partir de 2001 o Conselho Federal de Odontologia (CFO), homologou a especialidade para Pacientes com Necessidades Especiais (PNE), proporcionando aos Cirurgiões Dentista e acadêmicos de Odontologia capacitálos ao atendimento de pessoas que necessitam de cuidados odontológicos especiais, durante toda a vida ou por um período. Desta forma o objetivo dessa pesquisa é conhecer as dificuldades dos alunos de uma clínica escola odontológica nos atendimentos de pacientes com necessidades especiais, por meio do questionário. Foi realizada a aplicação de um questionário aos graduandos do $9^{\circ}$ e $10^{\circ}$ semestre da Faculdade de Odontologia $(n=60)$, com questões referentes ao atendimento odontológico e suas dificuldades sobre os PNE. O medo de machucar o paciente, a insegurança e o medo de mordedura foram as principais dificuldades apontadas por estudantes de Odontologia no atendimento de pacientes com necessidade especiais.
\end{abstract}

Descritores: Odontologia para pessoas com deficiência.dificuldade.assistência odontológica.

\section{Challenges in the care of patients with special needs in a school clinic}

\begin{abstract}
A large number of patients with disabilities that can be accessed by dental care." Meeting the demand requires a greater professional preparation, requiring a more humanized service. Since 2001, the Federal Council of Dentistry (CFO) has approved a specialty for Patients with Special Needs (PNE), providing Dental Surgeons and Dentists with Capacitors for the Care of Persons with Dental Care throughout their Life. or for a period. In this way, the research was carried out with the objective of sending the patients to a dental clinic in the care of patients with special needs, through the questionnaire. A questionnaire was applied to graduates of the 9th and 10th semester of the Faculty of Dentistry $(n=60)$, with emphasis on dental care and their difficulties on PNE.Conclusão: The fear of hurting the patient, insecurity and fear of the firsted cases in the portability of odontology in patients with special needs patients.
\end{abstract}

Descriptors: dentistry for people with disabilities. difficulty. dental care.

\footnotetext{
${ }^{1}$ Graduanda do curso de Odontologia da Faculdade Independente do Nordeste.

Contato: biiacabarros@gmail.com

${ }^{2}$ Especialista em Ortodontia. Especialista em saúde Pública e Docente do curso de Odontologia da Faculdade Independente do Nordeste.
}

919 Id on Line Rev. Mult. Psic. V.12, N. 42, p. 919-932, 2018 - ISSN 1981-1179 Edição eletrônica em http://idonline.emnuvens.com.br/id 


\section{Introdução}

De acordo com dados estimados em países desenvolvidos existe aproximadamente cerca de 500 milhões de indivíduos com deficiência em todo o mundo. De acordo com o Censo demográfico de 2000 (IBGE), 25 milhões de brasileiros, ou seja, 14,5\% da população possuem algum tipo de deficiência. A OMS (Organização Mundial da Saúde) avalia que a prevalência das deficiências seja de uma pessoa a cada dez, e afirma que desse total de deficientes, mais de dois terços não recebem nenhum tipo de assistência odontológica (PECORARO ;FERREIRA; MAIA 2017; COSTA;BONA,2013).

Desde 2001, com a Resolução 22/2001 do Conselho Federal de Odontologia (CFO), validou como especialidade, a Odontologia para Pacientes com Necessidades Especiais (PNEs). Propiciando ao profissional a capacidade em realizar o atendimento dos diferentes tipos de comprometimento (físico, intelectual, sensorial, orgânico, social e/ou comportamental) incluindo deficientes sensoriais(COSTA;BONA,2013).

O termo "pacientes com necessidades especiais" é utilizado para caracterizar indivíduos que não apresentam os padrões considerados normais para a sociedade, devido possuírem um desvio da normalidade de ordem física, mental, sensorial, comportamental e/ou de crescimento, carecem receber atenção diferenciado por um período ou durante a vida toda(GOMES et al., 2009;DOMINGUES et al., 2015).

O ensino odontológico dá um maior destaque ao paciente "normal" deixando de propiciar ao aluno os manejos necessários para o atendimento a pessoas com deficiência. Encontram-se alguns cursos que habilitam os alunos para realizar o atendimento de PNEs, concebendo um olhar diferenciado durante as práticas clínicas permitindo que os profissionais apreciem as necessidades desses indivíduos como um todo, prestem assistência e tratem com igualdade. (BARROS; DOS ANJOS; DOS SANTOS, 2013).

Os alunos necessitam de entusiasmo e co-participação para agregar aos serviços Odontológicos, tratando esses pacientes de uma maneira mais humanizada, motivados a participar ativamente dos serviços que tratam desses pacientes, percebendo em primeiro lugar o paciente e depois a deficiência. Com a finalidade de identificar e tratar os problemas físicos, sabendo escutar, perceber e interpretar o olhar do paciente, como sentimentos, mímicas e atitudes(AMARAL; Aquotte;Parizi;Oliveira, 2015). 
Existem pacientes com necessidades especiais que possuem problemas sistêmicos e psicológicos que necessitam de um acompanhamento médico constante. No entanto para realizar o atendimento odontológico, que implica na utilização de anestésicos, medicamentos, a classe medica expõem barreiras ao cuidado e contra-indicações das práticas odontológicas exigindo uma abordagem centrada nas decisões de tratamento(QUEEN et al., 2016).

O trabalho Odontológico deve ser bem preparado para atender os pacientes com necessidades especiais que acabam sendo pessoas vulneráveis, geralmente inclui fatores como falta de rede de apoio, desvantagem econômica, falta de assistência em atividades diárias. Refletindo os resultados no cuidado a saúde bucal (DOLAN et al., 2013). Estudantes que estão na graduação de Odontologia precisam ampliar seu conhecimento teórico e prático, dedicando, planejando e executando seu plano de tratamento de forma ética e honesta (FERREIRA et al., 2017).

Para realizar os atendimentos odontológicos o manejo do Cirurgião Dentista deve ser preciso para não ocorrer perturbações e agitações no consultório. Todavia esse conhecimento prático é imprescindível na etapa da graduação para efetivar o atendimento. O contato prévio com o paciente com necessidades especiais gera uma nova experiência que será acumulada na graduação até a etapa profissional, evitando a falta de competência para orientar os pais e responsáveis por estes pacientes (DE ANDRADE; ELEUTÉIO, 2015).

Diante do exposto, torna-se relevante este estudo para os acadêmicos do curso de Odontologia, com intuito de gerar uma percepção maior dessa especialidade tão pouco procurada e vivenciada pelos alunos, conhecendo as dificuldades dos alunos de uma clínica escola Odontológica nos atendimentos de pacientes com necessidades especiais.

\section{Metodologia}

A pesquisa teve início após aprovação do Comitê de Ética em pesquisa, segundo a resolução vigente para Ética em Pesquisa em Seres Humanos no 466/12 do Conselho Nacional de Saúde (Ministério da saúde, DF) (CAAE: 86597218.6.0000.5578, n. do parecer: 2.593.668). 
A pesquisa foi realizada através de questionário por estudantes do Curso Odontologia da Instituição de Ensino Superior Privada. Os participantes englobarão os alunos que já cursaram ou estão cursando a Disciplina de Clínica Integrada IV(Clínica de pacientes especiais). Totalizando uma quantidade de 60 indivíduos para responderem ao questionário constituído por 10 questões. Universalizou-se, como critério de inclusão da pesquisa todos os indivíduos que leram, concordaram e assinaram o Termo de Consentimento Livre Esclarecido (TCLE). Alunos da Instituição de Ensino Superior Privada graduando no curso de Odontologia, após terem concluído ou estejam cursando a disciplina do componente curricular, Clínica Integrada IV, disciplina que. Os critérios de exclusão são: Acadêmicos que não fazem parte da Instituição de Ensino Superior Privada, que não foram matriculados na disciplina e se não concordarem a assinar o Termo de Consentimento Livre Esclarecido (TCLE).

O questionário será realizado em um ambulatório da clínica Odontológica e os alunos serão chamados individualmente de forma aleatória. O pesquisador realizou pessoalmente a entrega do documento, sendo o mesmo respondido pelo participante. Ressaltando que o pesquisador não teve nenhuma interferência durante o preenchimento do questionário.

Para concluir foi realizada análise estatística na qual os resultados foram expressos como freqüências absolutas e relativas. As associações da principal dificuldade e grau de dificuldade com as questões relacionadas ao atendimento de pacientes com necessidades especiais foram verificadas por meio do teste exato de Fisher. O nível de significância adotado foi de $5 \%$ ( $\alpha=$ $0,05)$

Os dados foram tabulados e analisados no IBM SPSS Statistics for Windows (IBM SPSS. 21.0, 2012, Armonk, NY: IBM Corp.). 


\section{Resultados}

Tabela 1. Frequências absolutas e relativas das respostas dos estudantes de odontologia em relação ao atendimento de pacientes com necessidades especiais.

\begin{tabular}{|c|c|c|}
\hline Questões & $\mathrm{n}$ & $\%$ \\
\hline \multicolumn{3}{|c|}{$\begin{array}{l}\text { Em sua opinião a instituição na qual estuda proporciona infra estrutura para atendimento de } \\
\text { pacientes especiais? }\end{array}$} \\
\hline Sim & 37 & 61,7 \\
\hline Não & 23 & 38,3 \\
\hline \multicolumn{3}{|c|}{$\begin{array}{l}\text { Quais os procedimentos mais comuns realizados na clínica-escola aos pacientes com necessidades } \\
\text { especiais? }\end{array}$} \\
\hline Restauração & 57 & 95 \\
\hline Exodontia & 3 & 5 \\
\hline \multicolumn{3}{|c|}{ O paciente precisou de condicionamento durante os atendimentos? } \\
\hline Sim & 41 & 68,3 \\
\hline Não & 19 & 31,7 \\
\hline \multicolumn{3}{|c|}{ Já realizou contenção física nos pacientes com necessidades especiais? } \\
\hline Sim & 20 & 33,3 \\
\hline Não & 40 & 66,7 \\
\hline \multicolumn{3}{|c|}{ Seu conhecimento sobre as interações medicamentosas se enquadra em: } \\
\hline Pouco & 17 & 28,3 \\
\hline Normal/razoável & 42 & 70,0 \\
\hline Muito & 1 & 1,7 \\
\hline \multicolumn{3}{|c|}{ Qual seu interesse aos cursos de aperfeiçoamento e especialização em PNE após a graduação? } \\
\hline Não tenho interesse & 33 & 55,0 \\
\hline Tenho interesse & 27 & 45,0 \\
\hline \multicolumn{3}{|c|}{ Seu primeiro contato com um PNE foi na clínica-escola? } \\
\hline Sim & 41 & 68,3 \\
\hline Não & 19 & 31,7 \\
\hline \multicolumn{3}{|c|}{ Qual sua avaliação da condição de saúde bucal de pacientes PNE? } \\
\hline Ruim & 10 & 16,7 \\
\hline Regular/razoável & 46 & 76,7 \\
\hline Boa & 4 & 6,7 \\
\hline
\end{tabular}

PNE, portadores de necessidade especiais.

Na Figura 1 é apresentada a distribuição dos estudantes de odontologia, de acordo com a principal dificuldade no atendimento de pacientes com necessidades especiais. Observa-se que o medo de machucar o paciente, seguido por insegurança e medo de mordedura foram as dificuldades mais apontadas no atendimento de pacientes com necessidades especiais. 


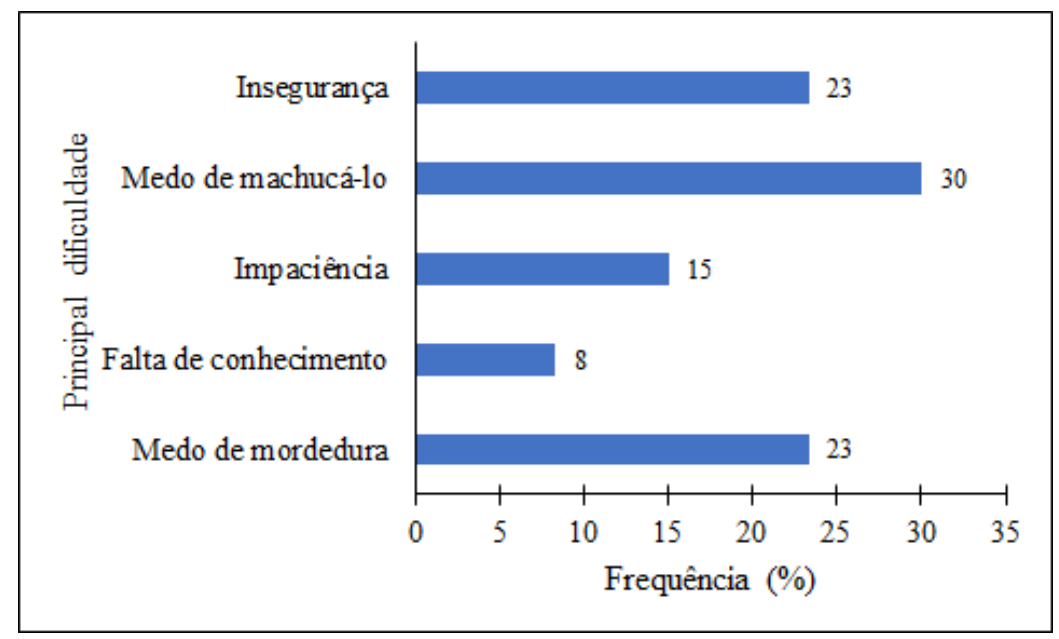

Figura 1. Principal dificuldade dos estudantes de odontologia no atendimento de pacientes com necessidades especiais.

Na Figura 2 é apresentada a distribuição dos estudantes de odontologia, de acordo com o grau de dificuldade no primeiro atendimento de pacientes com necessidades especiais. A maior parte dos estudantes considerou como normal ou razoável o grau de dificuldade no primeiro atendimento de pacientes com necessidades especiais.

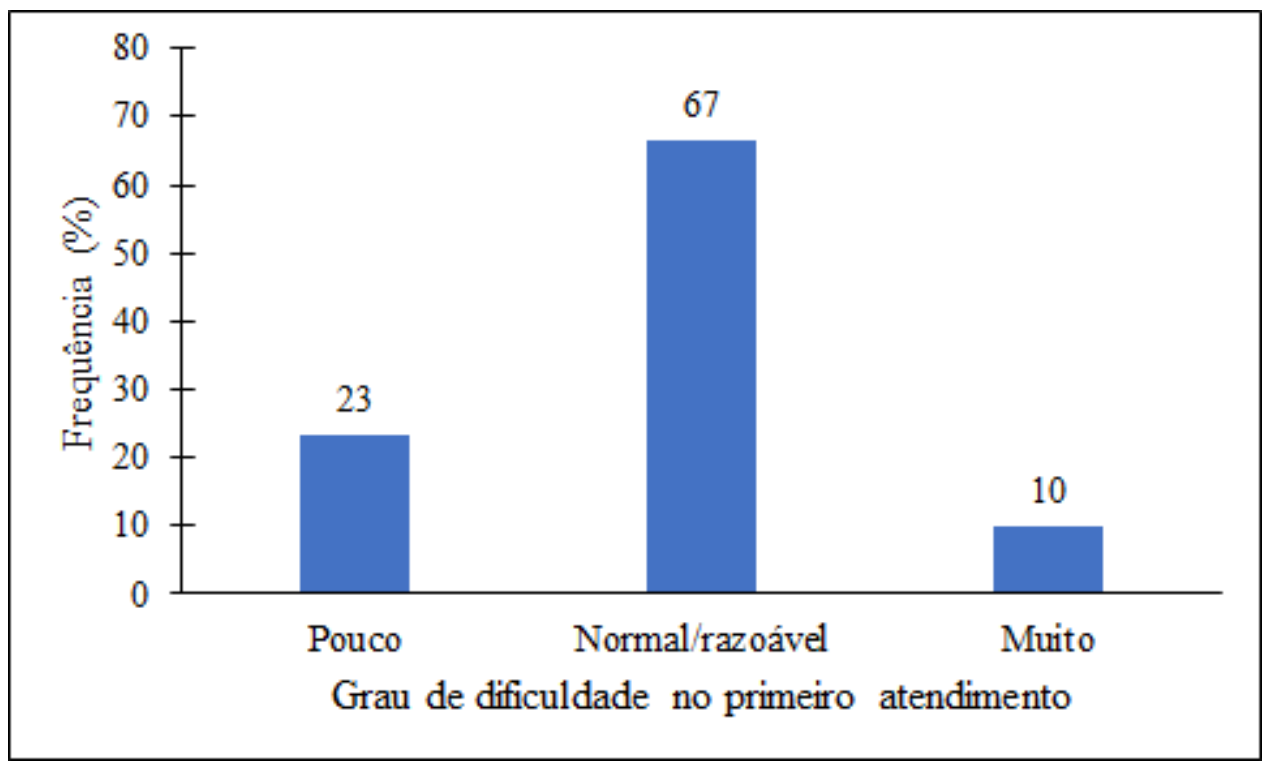

Figura2. Grau de dificuldade no primeiro atendimento dos estudantes de odontologia no atendimento de pacientes com necessidades especiais. 
Foram testadas associações entre a principal dificuldade no atendimento e as respostas dos estudantes de odontologia em relação ao atendimento de pacientes com necessidades especiais (Tabela 2). Os resultados das análises indicaram que houve associação apenas entre a principal dificuldade no atendimento e o interesse em cursos de aperfeiçoamento e especialização em PNE, sendo que os estudantes que indicaram não ter interesse nesses cursos apontaram maior frequência de impaciência e insegurança como principal de dificuldade, em relação aos estudantes que indicaram ter interesse; por outro lado, os estudantes que indicaram ter interesse nos cursos apontaram maior frequência de medo de mordedura e falta de conhecimento como principal de dificuldade, em relação aos estudantes que indicaram não ter interesse.

Tabela2. Principal dificuldade dos estudantes de odontologia, de acordo comas respostas em relação ao atendimento de pacientes com necessidades especiais.

\begin{tabular}{|c|c|c|c|c|c|c|}
\hline \multirow[b]{2}{*}{ Questões } & \multicolumn{5}{|c|}{ Principal dificuldade } & \multirow[b]{2}{*}{ *p-valor } \\
\hline & $\begin{array}{l}\text { Medo de } \\
\text { mordedura }\end{array}$ & $\begin{array}{c}\text { Falta de } \\
\text { conhecimento }\end{array}$ & Impaciência & $\begin{array}{c}\text { Medo de } \\
\text { machucá-lo }\end{array}$ & Insegurança & \\
\hline \multicolumn{7}{|c|}{ Em sua opinião a instituição na qual estuda proporciona infraestrutura para atendimento de pacientes especiais? } \\
\hline Sim & $10(27,0 \%)$ & $2(5,4 \%)$ & $3(8,1 \%)$ & $15(40,5 \%)$ & $7(18,9 \%)$ & \multirow[b]{2}{*}{0,056} \\
\hline Não & $4(17,4)$ & $3(13,0 \%)$ & $6(26,1 \%)$ & $3(13,0 \%)$ & $7(30,4 \%)$ & \\
\hline \multicolumn{7}{|c|}{ Quais os procedimentos mais comuns realizados na clínica-escola aos pacientes com necessidades especiais? } \\
\hline Restauração & $12(21,1 \%)$ & $5(8,8 \%)$ & $9(15,8 \%)$ & $18(31,6 \%)$ & $13(22,8 \%)$ & \multirow{2}{*}{0,452} \\
\hline Exodontia & $2(66,7 \%)$ & $0(0,0 \%)$ & $0(0,0 \%)$ & $0(0,0 \%)$ & $1(33,3 \%)$ & \\
\hline \multicolumn{7}{|c|}{ O paciente precisou de condicionamento durante os atendimentos? } \\
\hline Sim & $10(24,4 \%)$ & $4(9,8 \%)$ & $4(9,8 \%)$ & $14(34,1 \%)$ & $9(22,0 \%)$ & \multirow{2}{*}{0,502} \\
\hline Não & $4(21,1 \%)$ & $1(5,3 \%)$ & $5(26,3 \%)$ & $4(21,1 \%)$ & $5(26,3 \%)$ & \\
\hline \multicolumn{7}{|c|}{ Já realizou contenção física nos pacientes com necessidades especiais? } \\
\hline Sim & $7(35,0 \%)$ & $3(15,0 \%)$ & $1(5,0 \%)$ & $5(25,0 \%)$ & $4(20,0 \%)$ & \multirow{2}{*}{0,229} \\
\hline Não & $7(17,5 \%)$ & $2(5,0 \%)$ & $8(20,0 \%)$ & $13(32,5 \%)$ & $10(25,0 \%)$ & \\
\hline \multicolumn{7}{|c|}{ Seu conhecimento sobre as interações medicamentosas se enquadra em: } \\
\hline Pouco & $8(47,1 \%)$ & $1(5,9 \%)$ & $1(5,9 \%)$ & $3(17,6 \%)$ & $4(23,5 \%)$ & \multirow{3}{*}{0,105} \\
\hline Normal/razoável & $6(14,3 \%)$ & $4(9,5 \%)$ & $8(19,0 \%)$ & $15(35,7 \%)$ & $9(21,4 \%)$ & \\
\hline Muito & $0(0,0 \%)$ & $0(0,0 \%)$ & $0(0,0 \%)$ & $0(0,0 \%)$ & $1(100,0 \%)$ & \\
\hline \multicolumn{7}{|c|}{ Qual seu interesse aos cursos de aperfeiçoamento e especialização em PNE após a graduação? } \\
\hline Não tenho interesse & $5(15,2 \%)$ & $1(3,0 \%)$ & $8(24,2 \%)$ & $9(27,3 \%)$ & $10(30,3 \%)$ & \multirow{2}{*}{$\mathbf{0 , 0 3 2}$} \\
\hline Tenho interesse & $9(33,3 \%)$ & $4(14,8 \%)$ & $1(3,7 \%)$ & $9(33,3 \%)$ & $4(14,8 \%)$ & \\
\hline \multicolumn{7}{|c|}{ Seu primeiro contato com um PNE foi na clínica-escola? } \\
\hline Sim & $9(22,0 \%)$ & $2(4,9 \%)$ & $7(17,1 \%)$ & $13(31,7 \%)$ & $10(24,4 \%)$ & \multirow{2}{*}{0,686} \\
\hline Não & $5(26,3 \%)$ & $3(15,8 \%)$ & $2(10,5 \%)$ & $5(26,3 \%)$ & $4(21,1 \%)$ & \\
\hline \multicolumn{7}{|c|}{ Qual sua avaliação da condição de saúde bucal de pacientes PNE? } \\
\hline Ruim & $2(20,0 \%)$ & $1(10,0 \%)$ & $1(10,0 \%)$ & $3(30,0 \%)$ & $3(30,0 \%)$ & \multirow{3}{*}{0,872} \\
\hline Regular/razoável & $11(23,9 \%)$ & $4(8,7 \%)$ & $6(13,0 \%)$ & $14(30,4 \%)$ & $11(23,9 \%)$ & \\
\hline Boa & $1(25,0 \%)$ & $0(0,0 \%)$ & $2(50,0 \%)$ & $1(25,0 \%)$ & $0(0,0 \%)$ & \\
\hline
\end{tabular}

PNE, portadores de necessidade especiais.* Teste exato de Fisher. 
Também foram testadas associações entre o grau de dificuldade no primeiro atendimento e as respostas dos estudantes de odontologia em relação ao atendimento de pacientes com necessidades especiais (Tabela 3). Os resultados das análises indicaram que não houve associação entre o grau de dificuldade no primeiro atendimento e as questões avaliadas.

Tabela3. Grau de dificuldade no primeiro atendimento, de acordo com as respostas em relação ao atendimento de pacientes com necessidades especiais.

\begin{tabular}{|c|c|c|}
\hline & Grau c & ldade no primeir \\
\hline Questōes & Pouco & Normal/razoável \\
\hline
\end{tabular}

Em sua opinião a instituição na qual estuda proporciona infra estrutura para atendimento de pacientes especiais?
Sim
$9(24,3 \%)$
$27(73,0 \%)$
Não
$5(21,7 \%)$
$13(56,5 \%)$
$1(2,7 \%)$
$5(21,7 \%)$
0,076

Quais os procedimentos mais comuns realizados na clínica-escola aos pacientes com necessidades especiais?
Restauração
$13(22,8 \%)$
$38(66,7 \%)$
$2(66,7 \%)$
$6(10,5 \%)$
$1(33,3 \%)$
$0(0,0 \%)$
1,000

O paciente precisou de condicionamento durante os atendimentos?
Sim
$7(17,1 \%)$
$28(68,3 \%)$
Não
$7(36,8 \%)$
$12(63,2 \%)$
$6(14,6 \%)$
$0(0,0 \%)$
0,093

Já realizou contenção física nos pacientes com necessidades especiais?
Sim
$4(20,0 \%)$
$12(60,0 \%)$
$4(20,0 \%)$
Não
$10(25,0 \%)$
$28(70,0 \%)$
$2(5,0 \%)$
0,249

Seu conhecimento sobre as interações medicamentosas se enquadra em:
Pouco
$4(23,5 \%)$
$11(64,7 \%)$
$2(11,8 \%)$
Normal/razoável
$10(23,8 \%)$
$29(69,0 \%)$
$3(7,1 \%)$
0,258
Muito
$0(0,0 \%)$
$0(0,0 \%)$
$1(100,0 \%)$

Qual seu interesse aos cursos de aperfeiçoamento e especialização em PNE após a graduação?
Não tenho interesse
$8(24,2 \%)$
$22(66,7 \%)$
$3(9,1 \%)$
Tenho interesse
$6(22,2 \%)$
$18(66,7 \%)$
$3(11,1 \%)$
1,000

Seu primeiro contato com um PNE foi na clínica-escola?
Sim
$9(22,0 \%)$
$28(68,3 \%)$
$12(63,2 \%)$
$4(9,8 \%)$
$5(26,3 \%)$
$2(10,5 \%)$
0,912

Qual sua avaliação da condição de saúde bucal de pacientes PNE?

\begin{tabular}{lcccc} 
Ruim & $1(10,0 \%)$ & $8(80,0 \%)$ & $1(10,0 \%)$ & \\
Regular/razoável & $10(21,7 \%)$ & $31(67,4 \%)$ & $5(10,9 \%)$ & 0,187 \\
Boa & $3(75,0 \%)$ & $1(25,0 \%)$ & $0(0,0 \%)$ & \\
\hline
\end{tabular}

PNE, portadores de necessidade especiais. * Teste exato de Fisher. 


\section{Discussão}

A Odontologia para Pacientes com Necessidades Especiais (PNE) tem sido evidenciada na literatura há algum tempo. Com os avanços tecnológicos principalmente na área da saúde a expectativa de vida de PNE aumentou, e a vinda desses pacientes ao consultório odontológico tem sido cada vez mais frequente( LAWRENCE et al., 2014).

O presente estudo analisou as dificuldades dos alunos no atendimento odontológico em pacientes especiais, os participantes que foram contemplados pelas abordagens do questionário totalizaram uma amostra de 60 indivíduos, sendo graduando em Odontologia de uma Instituição privada de ensino superior, que já haviam tido contato com a disciplina de PNE.

A literatura salienta que é necessária a humanização nos atendimentos odontológicos, mais contato e acolhimento para que haja maior colaboração do paciente, podendo diminuir ou eliminar um evento desfavorável presente ${ }^{12}$. Entretanto não é isso que se vê na realidade. Diante da análise apresentada neste trabalho 68,3\% dos acadêmicos precisou realizar condicionamento durante o atendimento. Segundo Moraes et al.,(2006), a preparação de alunos para lidar com pacientes especiais vai além da formação técnica, sendo necessário ter uma sensibilização e buscar manobras de atendimento na clínica.

Quando se trata das associações testadas entre a principal dificuldade no atendimento e o interesse em cursos de aperfeiçoamento e especialização em PNE, analisou associação nas mesmas. Contudo o exercício profissional da Odontologia vem mudando sua vertente nos tempos atuais, existindo a concorrência de proporcionar oferta mediante a procura. Observa-se um crescimento de cursos de especialização, no entanto devido as dificuldades enfrentadas nos atendimentos e a concentração em certas áreas, os acadêmicos ao saírem da universidade acabam escolhendo a especialidade que possui maior facilidade em atuar (LINO QUEIROZ; ARAUJO ,2006; PEREIRA et al., 2010). A maioria dos estudantes pesquisados (55\%) respondeu "não tenho interesse" no curso de especialização.

Os procedimentos mais comuns de acordo com a pesquisa são restaurações e exodontias. Isso se deve, segundo estudos, à qualidade da higiene oral por meio dos pacientes com necessidades especiais, que por vezes possuem dificuldades/limitações em realizar uma boa higiene oral e assim, consequentemente, há maiores chances de desenvolver problemas orais, como cárie dentária, dor e desconforto.(VELLAPPALLY et al., 2014). 
Convém salientar, que este estudo evidenciou que $80 \%$ dos entrevistados consideraram regular/razoável a avaliação de saúde bucal dos PNE. Normalmente esses pacientes apresentam índices de saúde bucal mais precários que a população de maneira geral (SOLANKI;GUPTA; ARYA,2014; HARTWIG et al., 2015).Isso se deve pelo fato deste indivíduos possuírem dificuldades em sua higienização, e dependem da ajuda dos seus cuidadores que, em alguns casos, também não tem o conhecimento para realizar de forma correta a higiene oral dessas pessoas(AMEER et al., 2012; ALTUN et al.,2014).

Com os resultados, observa-se que o preparo dos graduandos de acordo com o grau de dificuldade no primeiro atendimento é considerado normal/razoável. Segundo JACOMINE et al.,(2018), os acadêmicos que cursaram a disciplina do componente curricular, necessitam de mais ações, como projetos extra curriculares proporcionando uma melhor vivências e experiência para realizar os atendimentos Odontológico.

A análise estatística tornou notório, que os acadêmicos tiveram o primeiro contato com pacientes especiais na clínica escola. Os sentimentos mais prevalentes encontrados na pesquisa foram insegurança (23\%), medo de machucá-lo (30\%), impaciência (15\%), falta de conhecimento (8\%), medo de mordedura (23\%). Para que essa situação se transforme e se reduzam as vivências negativas no atendimento se faz importante um desenvolvimento multidisciplinar, em que a partir do momento no qual serão divididas as experiências vivenciadas e compartilhada as emoções, haverá contribuição para atenção prestada aos pacientes e seus familiares(COYLE; SAUNDERSON;FREEMAN, 2004;BORGES et al, 2015).

\section{Conclusão}

Com base nos resultados é possível concluir que:

- O medo de machucar o paciente, a insegurança e o medo de mordedura foram as principais dificuldades apontadas por estudantes de odontologia no atendimento de pacientes com necessidades especiais; 
- As maiorias dos estudantes de Odontologia não perceberam grau de dificuldade aumentado no atendimento de pacientes com necessidades especiais;

- A principal dificuldade no atendimento de pacientes com necessidades especiais variou em função do interesse dos estudantes em se atualizar e/ou especializar em atendimento de PNE. Os estudantes com interesse na formação continuada na área de PNE foram aqueles que, em relação aos seus pares, indicaram maior medo de mordedura e falta de conhecimento como principais dificuldades.

- O grau de dificuldade no primeiro atendimento de pacientes com necessidades especiais não foi influenciado pelos fatores relacionados ao atendimento avaliados neste estudo.

\section{Referências}

AMEER N, PALAPARTHI R, NEERUDUM M, PALAKURU SK, SINGAM HR, DURVASULA S. Oral hygiene and periodontal status of teenagers with special needs in the district of Nalgonda, India. Journal of Indian Society of Periodontology, v. 16, n. 3, p. 421, 2012. Disponivel em < https://www.ncbi.nlm.nih.gov/pmc/articles/PMC3498715/>. Acesso em 24 out.2018.

ALTUN C, GUVEN G, AKGUN OM, AKKURT MD, BASAK F, AKBULUT E, Ceyhan et al. Oral health status of disabled individuals attending special schools. European journal of dentistry, $\quad$ v. 4, n. 4, p. 361, 2010. Disponivel em:< https://www.ncbi.nlm.nih.gov/pmc/articles/PMC2948746> Acesso em 24 out.2018.

AMARAL COF, AQUOTTE APDC, AQUOTTE LDC, PARIZI AGS, OLIVEIRA AD.. Avaliação das expectativas e sentimentos de alunos de odontologia frente ao atendimento de pacientes com necessidades especiais. Revista da Faculdade de Odontologia-UPF, v. 16, n. 2, 2015. Disponível em : < http://www.seer.upf.br/index.php/rfo/article/view/1528/1349> Acesso em 24 out.2018.

BARROS ALO, HORA IADA, SANTOS MTBR. Análise do perfil do profissional cirurgiãodentista que atende pacientes com necessidades especiais. Revista Brasileira de Pesquisa em Saúde/Brazilian Journal of Health Research, v. 15, n. 3, 2013. Disponível em : <http://www.portaldepublicacoes.ufes.br/RBPS/article/viewFile/6324/4658. Acesso em 24 out.2018. 
BORGES, MM. OPINIÕES DOS ALUNOS DE ODONTOLOGIA SOBRE O ATENDIMENTO A PACIENTES COM NECESSIDADES ESPECIAIS. Horizonte $\begin{array}{lllllll}\text { Científico, } & \text { v. } & 9, & \text { n. } & \text { 2,2015.Disponível } & \text { em } & :\end{array}$ http://www.seer.ufu.br/index.php/horizontecientifico/article/view/22255> Acesso em 24 out.2018.

COSTA AAI, BONA AD. Atendimento odontológico de pacientes surdo-cegos: enfrentando desafios. RFO UPF, v. 18, n. 1, p. 107-111, 2013. Disponível em :< http://revodonto.bvsalud.org/scielo.php?pid=S1413-

40122013000100018\&script=sci_arttext\&tlng=pt $>$.Acesso em 24 out.2018.

COYLE C, SAUNDERSON W, FREEMAN R. Dental students, social policy students and learning disability: do differing attitudes exist?. European Journal of Dental Education, v. 8, n. 3, p. 133-139, 2004.Disponivel em < https://onlinelibrary.wiley.com/doi/abs/10.1111/j.16000579.2004.00343.x> Acesso em 24 out.2018.

DE ANDRADE, APP, ELEUTÉIO, ASDLE. Pacientes portadores de necessidades especiais: abordagem odontológica e anestesia geral. Revista Brasileira de Odontologia, v. 72, n. 1/2, p.

66 ,

2016.

Disponível em : $<$ http://revista.aborj.org.br/index.php/rbo/article/view/616> Acesso em 24 out.2018.

DOMINGUES NB, AYRES KCM, MARIUSSO MR, ZUANON ACC, GIRO EMA. Caracterização dos pacientes e procedimentos executados no serviço de atendimento a pacientes com necessidades especiais da Faculdade de Odontologia de AraraquaraUNESP. Rev Odontol UNESP, v. 44, n. 6, p. 345-350, 2015. Disponível em :< http://www.scielo.br/pdf/rounesp/2015nahead/1807-2577-rounesp-1807-25770015.pdf>

Acesso em 24 out.2018.

DOLAN, Teresa A. Professional education to meet the oral health needs of older adults and persons with disabilities. Special Care in Dentistry, v. 33, n. 4, p. 190-197, 2013. Disponível em :< https://onlinelibrary.wiley.com/doi/abs/10.1111/scd.12013> Acesso em 24 out.2018.

FERREIRA SH, SUITA RA, RODRIGUES PH, KRAMER PF. Percepção de estudantes de graduação em Odontologia frente ao atendimento de pessoas com deficiência. Revista da ABENO, v. 17, n. 1, p. 87-96, 2017.Disponível em $:<$ https://revabeno.emnuvens.com.br/revabeno/article/view/367> Acesso em 24 out.2018.

GUERRA, CT et al. Reflexões sobre o conceito de atendimento humanizado em Odontologia. Archives of Health Investigation, v. 3, n. 6, 2015.Disponivel em :< http://archhealthinvestigation.com.br/ArcHI/article/view/72> Acesso em 24 out.2018.

GOMES M et al. A percepção dos docentes do Curso de Odontologia da UFES em relação à necessidade de inclusão da disciplina denominada Atendimento Odontológico a Pacientes 
Portadores de Necessidades Especiais. Revista Brasileira de Pesquisa em Saúde/Brazilian Journal of Health Research, 2009.Disponível em http://www.publicacoes.ufes.br/RBPS/article/viewFile/446/310> Acesso em 24 out.2018.

HARTWIG, AD et al. Recursos e técnicas para a higiene bucal de pacientes com necessidades especiais. Revista da AcBO-ISSN 2316-7262, v. 4, n. 3, 2015.Disponível em:< http://www.rvacbo.com.br/ojs/index.php/ojs/article/view/272> Acesso em 24 out.2018.

JACOMINE, JC et al. Saúde bucal e Pacientes com Necessidades Especiais: percepções de graduandos em Odontologia da FOB-USP. Revista da ABENO, v. 18, n. 2, p. 45-54, 2018.Disponível em : < https://revabeno.emnuvens.com.br/revabeno/article/view/434> Acesso em

out.2018.

LAWRENCE H, SOUZA LP, GONÇALVES FL, SAINTRAIN MVL, VIEIRA APGF. Acesso à saúde bucal pública pelo paciente especial: a ótica do cirurgião-dentista. Revista Brasileira em Promoção da Saúde, v. 27, n. 2, p. 190-197, 2014. Disponível em:< http://periodicos.unifor.br/RBPS/article/view/2620> Acesso em 24 out.2018.

LINO RG, Queiroz MC, Araujo JEEA.MERCADO DE TRABALHO NA ODONTOLOGIA: CONTEXTUALIZAÇÃO E PERSPECTIVAS. e-RAC, v. 7, n. 1, 2018.Disponível em: < http://www.computacao.unitri.edu.br/erac/index.php/e-rac/article/view/1087> Acesso em 24 out.2018.

MORAES, ABA et al. Verbalizações de alunos de odontologia sobre a inclusão social de pessoas com deficiência. Psicologia em Estudo, 2006.Disponível em:< http://repositorio.unicamp.br/handle/REPOSIP/26597> Acesso em 24 out.2018.

MACEDO, LP et al. Atenção odontológica em pacientes com deficiências: a experiência do curso de Odontologia da ULBRA Canoas/RS. Stomatos, v. 16, n. 31, 2010. Disponível em:< http://www.redalyc.org/html/850/85018679011> Acesso em 24 out.2018.

PECORARO PVBF, FERREIRA M, MAIA MPDC, CONDÉ SP. Pacientes com deficiências: metodologia e prática de inclusão social na faculdade de odontologia de Valença/RJ. Revista Interdisciplinar de Direito, v. 10, n. 2, 2017. Disponível em $:<$ file:///C:/Users/Win7/Downloads/180-1-292-1-10-20171002\%20(8).pdf> Acesso em 24 out.2018.

QUEEN, AN. Evidence-based Dentistry and Its Role in Caring for Special Needs Patients. Dental Clinics, v. 60, n. 3, p. 605-611, 2016.Disponivel em :< https://www.dental.theclinics.com/article/S0011-8532(16)30013-1/abstract> Acesso em 24 out.2018. 
SOLANKI, J; GUPTA, S; ARYA, A. Dental caries and periodontal status of mentally handicapped institutilized children. Journal of clinical and diagnostic research: JCDR, v. 8, n. $7, \quad$ p. $\quad$ ZC25, 2014.Disponível em $\quad:<$ https://www.ncbi.nlm.nih.gov/pmc/articles/PMC4149138/> Acesso em 24 out.2018.

VELLAPPALLy S, GARDENS SJ, AL KHERAIF AA, KRISHNA M, BABU S, HASHEM $\mathrm{M}$. The prevalence of malocclusion and its association with dental caries among 12-18-yearold disabled adolescents. BMC oral health, v. 14, n. 1, p. 123, 2014.Disponível em :< https://bmcoralhealth.biomedcentral.com/articles/10.1186/1472-6831-14-123> Acesso em 24 out.2018.

\section{Como citar este artigo (Formato ABNT):}

BARROS, Bianca Cardoso; CUNHA, Daniela Porto da. Desafios no atendimento ao paciente portador de necessidades especiais em uma clínica escola. Id on Line Rev.Mult. Psic., 2018, vol.12, n.42, p. 919932. ISSN: 1981-1179.

Recebido: 25/10/2018;

Aceito: $26 / 10 / 2018$ 